\title{
Variation of the Energy Landscape of a Small Peptide under a Change from the ECEPP/2 Force Field to ECEPP /3
}

\author{
Frank Eisenmenger ${ }^{\dagger}$ and Ulrich H.E. Hansmann\# 円 \\ ${ }^{\dagger}$ Institute for Biochemistry, Medical Faculty (Charitè) \\ of the Humboldt University Berlin, 10115 Berlin, Germany \\ \# Swiss Center for Scientific Computing (SCSC), \\ Federal Institute of Technology (ETH) Zürich, 8092 Zürich, Switzerland
}

\begin{abstract}
The multicanonical ansatz is used to study variations in the energy landscape of a small peptide, Met-enkephalin, under a change from the ECEPP/2 force field to ECEPP/3. Local minima with energies up to $5 \mathrm{kcal} / \mathrm{mol}$ higher than the global minima are sampled and classified according to H-bridges and backbone angles. The distribution and relative weight for various temperatures of the minima are calculated and compared for the two force fields. We demonstrate that while there are small differences in the energy landscape our results at relevant temperatures are robust under changes between ECEPP/2 to ECEPP/3.
\end{abstract}

\footnotetext{
${ }^{1}$ New address: Institute for Molecular Science (IMS), Okazaki 444, Japan
} 


\section{Introduction}

While there is considerable progress in numerical simulation of peptides and proteins (for a recent review see, for instance, [1]), prediction of their low temperature conformations solely from first principles remains a formidable task. First, the interactions between the atoms in the molecules are described by force fields which depend in part on empirically determined parameters. It is still an open question which of the many force fields (AMBER [2], CHARMM [3], ECEPP [4] - [7], ...) is the optimal choice (or if they are sufficiently accurate at all). The other mayor problem is common to many important physical systems. At low temperatures the energy landscape of proteins is characterized by a multitude of local minima separated by high energy barriers. In simulations based on classical molecular dynamics or Monte Carlo techniques [8] these barriers can be seldom crossed. Hence, only small parts of phase space are sampled (in a finite number of Monte Carlo sweeps) and physical quantities cannot be calculated accurately. For this reason, the "native" conformation of a protein (which should correspond to the global minimum in free energy) is often identified with the lowest potential energy configuration. In this approximation entropic contributions are ignored, but it allows application of optimization techniques like simulated annealing [10], genetic algorithms [9] or Monte Carlo with Minimization [11] to tackle the protein folding problem. However, this approximation may be to crude. Especially for smaller peptides entropic contributions are expected to be important.

It is not always clear whether the limited quality of numerical results is due to insufficient simulation algorithms or inadequate force fields. Recent progress in the development of Monte Carlo techniques may change this situation. Simulations in generalized ensembles promise a much better sampling of the phase space. A numerical comparison of some of these new algorithms can be found in Ref. [12]. One of its more prominent exponents is the so called multicanonical approach of Berg and co-workers 13] who propose a weighting scheme which yield to a flat probability distribution in energy. Hence, all energies have equal weight and an one-dimensional random walk in energy space is carried out (when simulated with local updates) which ensures that the simulation will not 
get trapped in any local energy minimum. The Boltzmann distributions may be obtained for a given range of temperatures from one multicanonical simulation by re-weighting the states [14]. The method was exploited to model first-order phase transitions [13, 15] and spin-glass systems [16, 17, 18]. The prediction of peptide and protein three-dimensional structures with multicanonical algorithms was first addressed in Ref. [19 for Monte Carlo methods and in Ref. [20] for molecular dynamics. Subsequent works include the study of coil-globular transitions of a model protein [21], helix-coil transitions of amino acid homo-oligomers [22] and the conformational sampling of a constrained peptide [23].

In the current article we use the multicanonical technique to compare distribution and relative weight of the local minima at room temperature and below for the closely related ECEPP/2 and ECEPP/3 force field in the case of the small pentapeptide Met-enkephalin. ECEPP/3 deviates from the previous ECEPP/2 by slightly different parameters due to improvements in the experimental measurements. We want to study the influence of such small changes on the energy landscape and our observed quantities. This should show us how much numerical simulations are affected by experimental uncertainties in the parameters of the force fields.

\section{Methods}

\section{Multicanonical Algorithm}

Although the multicanonical approach is explained in detail elsewhere (see, for instance Refs. [16, 24]), we briefly summarize the idea and implementation of the method for completeness.

Simulations in the canonical ensemble weight each configuration with $w_{B}(E, \beta)=e^{-\beta E}$ and yield the usual Boltzmann probability density distribution of energies:

$$
P_{B}(E, T) \propto n(E) e^{-\beta E}
$$

where $n(E)$ is the density of states with energy $E$, and $\beta=\frac{1}{k_{B} T}$; with temperature $T$ and Boltzmann constant $k_{B}$.

On the other hand, in the multicanonical approach configurations with energy $E$ are 
updated with a weight:

$$
w_{m u}(E) \propto n^{-1}(E)=e^{-S(E)}
$$

where $S(E)$ is the microcanonical entropy. A uniform distribution of the energy

$$
P_{m u}(E)=n(E) w(E)=\text { const }
$$

is obtained from the simulation with these weight factors. In this way information is collected over the whole energy range and from a single simulation one can obtain the canonical distribution for a wide range of temperatures by the re-weighting techniques: 14

$$
P_{B}(T, E) \propto P_{m u}(E) w_{m u}^{-1} e^{-\beta E} .
$$

This allows one to calculate any thermodynamic quantity $\mathcal{O}$ by

$$
<\mathcal{O}>_{T}=\frac{\int d E \mathcal{O}(E) P_{B}(T, E)}{\int d E P_{B}(T, E)} .
$$

However, unlike in a canonical simulation the weights $w_{m u}(E)$ are not a priori known (in fact, knowledge of the exact weights is equivalent with solving the system), and one needs its estimators for a numerical simulation. One way to obtain these estimators is the following iterative procedure. Starting with an initial guess $w_{m u}^{0}(E)=e^{-\beta_{0} E}$, i.e., performing a canonical simulation at sufficient high temperature $T_{0}$, improved estima-

tors of the multicanonical weights are calculated from histograms $P_{m u}^{i-1}(E)$ of preceding simulations by

$$
w_{m u}^{i}(E)=\frac{w_{m u}^{i-1}(E)}{P^{i-1}(E)} .
$$

For details, see Ref. [22] and [24]. This method for calculating multicanonical weights is by no means unique and while it is quite general, it has the disadvantage that it requires a certain number of extra iterations which is not a priori known.

\section{Force-Fields}

In ECEPP [4]-[7] the potential energy function $E_{t o t}$ is given by the sum of the electrostatic term $E_{C}, 12-6$ Lennard-Jones term $E_{L J}$, and hydrogen-bond term $E_{H B}$ for all pairs of 
atoms in the peptide together with the torsion term $E_{\text {tor }}$ for all torsion angles:

$$
\begin{aligned}
E_{t o t} & =E_{C}+E_{L J}+E_{H B}+E_{t o r} \\
E_{C} & =\sum_{(i, j)} \frac{332 q_{i} q_{j}}{\epsilon r_{i j}} \\
E_{L J} & =\sum_{(i, j)}\left(\frac{A_{i j}}{r_{i j}^{12}}-\frac{B_{i j}}{r_{i j}^{6}}\right) \\
E_{H B} & =\sum_{(i, j)}\left(\frac{C_{i j}}{r_{i j}^{12}}-\frac{D_{i j}}{r_{i j}^{10}}\right) \\
E_{t o r} & =\sum_{l} U_{l}\left(1 \pm \cos \left(n_{l} \chi_{l}\right)\right)
\end{aligned}
$$

Here, $r_{i j}$ is the distance between the atoms $i$ and $j$, and $\chi_{l}$ is the torsion angle for the chemical bond $l$. In ECEPP bond lengths and bond angles (which are hard degrees of free-

dom) are fixed at experimental values and no out-of-plane deformation of peptide bonds is allowed leaving the dihedral angles $\phi, \psi, \omega$ and $\chi$ as independent variables. The various parameters $\left(q_{i}, A_{i j}, B_{i j}, C_{i j}, D_{i j}, U_{l}\right.$, and $\left.n_{l}\right)$ for the energy function were determined by a combination of a priori calculations and minimization of the potential energies of the crystal lattices of single amino acids. In the light of more recent experimental findings the standard geometry and some energy parameters for prolyl and hydroxyprolyl have been updated. Together with a re-calculation of partial atomic charges of mainchain atoms this led to a revision of the ECEPP/2 parameter set to ECEPP/3. In the original formulation of the ECEPP/2 parameter set, the net charges of both the $\mathrm{N}$ - and the C-terminal residues, added up to the desired net charge of the molecule. Now each terminal amino acid residue carries a total charge of $0 /+1 /-1$ at the $\mathrm{N}$ - or C-terminus, respectively. The correction avoids possible artifacts for systems with terminal groups of different types (e.g. one terminus charged, the other uncharged).

\section{Simulation and Technical Details}

To investigate changes in the energy landscape under small variations of the force field we have studied one of the simplest peptides, Met-enkephalin which has the amino acid sequence Tyr-Gly-Gly-Phe-Met. This peptide is convenient for our purpose, since for the potential energy function ECEPP/2 the lowest energy conformation is known [11, 25, 26] 
and local minima with energies not much higher than the global minimum were sampled and classified for this molecule by Braun and co-workers. 27 We compare our results with theirs and calculate in addition the relative weight of the local minima at various temperatures which is not possible with the method used by Braun et al.

For our simulations the backbone was terminated by a neutral $-\mathrm{NH}_{2}$ group at the N-terminus and a neutral $-\mathrm{COOH}$ group at the C-terminus as in the previous works of Met-enkephalin. For Met-enkephalin the two versions of ECEPP differ in the point charges for terminal groups which are listed in Tab. 1. The peptide-bond dihedral angles $\omega$ were fixed at the value $180^{\circ}$, leaving 19 dihedral angles as independent variables. 2 Interactions of the molecule with solvent molecules were neglected and the dielectric constant $\epsilon$ was set equal to 2. We used the program SMC [28] which was modified to accommodate the multicanonical ensemble.

The multicanonical weight factors were determined separately for both ECEPP/2 and ECEPP/3 by the iterative procedure described above. We needed 100,000 sweeps for their calculation. One MC sweep updates every dihedral angle of the molecule once. For each update of an angle a new randomly chosen value out of the interval $[-\pi, \pi]$ was proposed and the new configuration then accepted or rejected by the Metropolis criteria. All thermodynamic quantities were calculated for ECEPP/2 and ECEPP/3 separately from a production run of 200,000 MC sweeps following additional 10,000 sweeps for equilibration each. The energy after each sweep was stored for further analysis. In all cases, each simulation started from a completely random initial conformation ("Hot Start"), but in the case of ECEPP/2 we also checked our results for shorter runs against these with initial configuration of the known groundstate ("Cold Start"), and we found that the results are in agreement with those from random initial conformations. This suggests that thermal equilibrium has been attained in our simulations. This conjecture is further supported by Fig. 1, in which we show the timeseries of the energy for both

\footnotetext{
${ }^{2}$ We fixed these hard degrees of freedom to reduce the number of variables. It would be more rigorous to allow for bending of the peptide angles. Similarly, one could argue that the fixed geometry of ECEPP should be replaced by a flexible one (as in other force fields), for instance by including Fixman potentials. However, in this article we are mostly interested in comparing two variants of a force field, not in reproducing experimentally found structures by numerical simulation, which allow us to choose a less rigorous approach.
} 
production runs. In the course of the simulation, a $1 \mathrm{~d}$ random walk in the energy between low energy states and high energy states is performed as one would expect for local updates from the definition of the multicanonical ensemble. It is evident that low energy states which are separated in the timeseries by high energy states (which correspond to high temperature states) are uncorrelated. The number of such "tunnel" events is therefore a lower limit for the number of independent low energy states visited in the simulation.

In order to investigate the energy landscape of our peptide we not only stored the energy of the actual conformation after each MC sweep, but also minimized a copy of this conformation if its energy was less than a certain limit, $0 \mathrm{kcal} / \mathrm{mol}$. While this limit is somehow arbitrary, our experience shows that it allows a rough distinction between a "low temperature region" and a "high temperature region" (it is the expectation value of Energy at $T=300 K$ where the specific heat has its maximum). From the minimization process we receive a local minimum conformation. Configurations which yield the same local minima belong to the same valley in the energy landscape and are therefore related to each other. Since we are mainly interested in local minima not too far away from the global minimum and to save disc space, we introduced a second limit. Only if the energy of the minimized configuration was below $-6 \mathrm{kcal} / \mathrm{mol}(\approx 5 \mathrm{kcal} / \mathrm{mol}$ above the global minimum) it was stored for further analysis. In Tab. 2 we summarize the absolute number of local minima sampled in this way for both variants of the ECEPP force field.

While the set of configurations we received from our production runs are all local minima, due to limitations of the minimizer (a Newton-Raphson variant) they are not necessarily the lowest configurations associated with a certain valley in the energy landscape. Each valley is in itself rough and consists of subvalleys separated by energy barriers. Since we are not interested in the microscopic details of the energy landscape we further clustered our local minima and identified each cluster by its member with the lowest energy. In this way, only a small number (less than 5) of groups remains at the end of our clustering by which the overwhelming number of local minima is classified.

We describe now briefly our clustering technique. In a first step we sorted all minimized conformers according to their energy. In a second step, we looked for identical conformers. Two conformers were regarded as identical if they differ in no angle more than $1^{\circ}$ (taking 
symmetries in account) and in their energies less than $0.0001 \mathrm{kcal} / \mathrm{mol}$. For further clustering, we kept only the non-identical conformations. Their number which is also shown in Tab. 2 is our estimate for the total number of local minima with energy below $-6 \mathrm{kcal} / \mathrm{mol}$. It is interesting to observe that the number of non-identical conformers sampled in the multicanonical runs is similar for each choice of force field while the absolute number of sampled local minima varies much more (see Tab. 2). In a third step, using all backbone angles, the conformer (from our remaining set of non-identical local minima conformations) with the lowest energy is compared to all other with higher energies, according to differences in their backbone dihedrals. The conformers having all their angles within $10^{\circ}$ in comparison with the first one, with respect to symmetry, are put into the same cluster, which is represented by the first conformer. This procedure is repeated for the next conformer with higher energy, which was not counted in the first cluster by comparing its angles with all the other remaining "free" conformers. In this way, we are left with 160 clusters for ECEPP/2 and 571 clusters for ECEPP/3. In the final step these clusters were classified into few groups using similarities in hydrogen bonding and the significant dihedrals, necessary to preserve the characteristic hydrogen bonds. The final groups which should represent the main valleys in the energy landscape of our peptide are characterized by their lowest-energy conformer.

In Fig. $2 \mathrm{a}$ and $2 \mathrm{~b}$ we display how in the course of our simulations different valleys of the energy landscape (characterized by its corresponding group number) are visited. We show only that times when the simulation visit one of the 4 (3) most important clusters for ECEPP/2 (ECEPP/3) and no plot symbol means that the simulation did not visit one of these valleys. As one can see there are different slices in which the multicanonical simulation moved only inside of a valley separated by jumps between different valleys. Again the number of these slices will give a lower limit on the number of independent low energy states visited in the simulation.

\section{Results and Discussion}

Using the techniques described above we were able for each choice of the force field to classify the large number of local minimum conformations sampled in our multicanonical 
runs into few groups which represent more than $80 \%$ of the sampled local minimum conformations and which correspond to the important valleys in the energy landscape of Met-enkephalin. In Fig. 3a and Fig. 3b the dominant groups for both variants of the ECEPP force field are shown. In addition, we present in Tab. 3 the dihedral angles for the global minimum conformations obtained by our method. These structures are also shown in Fig. 4a and Fig. 4b.

The global minimum for ECEPP/3 has characteristic hydrogen bond of the NH-group of Phe(4) with the carbonyl group of Try(1) resulting in a $\beta$-bend between the first and fourth residue. To preserve this hydrogen bond only the backbone angles of Gly $(2)$ and Gly(3) have to be conserved and therefore one finds a large number of variants differing from the global minimum stucture in the other backbone and sidechain dihedrals in this group (which we call group "1"). The second group ("2") is characterized by the local minimum configuration with the second lowest energy in the ECEPP/3 force field. This configuration has two possible hydrogen bonds between $\mathrm{N}-\mathrm{H}$ and $\mathrm{C}=\mathrm{O}$ groups of the $\operatorname{Gly}(2)$ and $\operatorname{Met}(5)$ residue forming a II'-type $\beta$-turn between the second and last residue. Since more backbone dihedrals have to be conserved to preserve the two hydrogen bonds the configurations of this group vary much less than in the case of the previous group. Despite their differences (see Figs. 3 and 4) the lowest energy exponent of both groups differs by only $0.04 \mathrm{kcal} / \mathrm{mol}$ in potential energy, so that one can regard the global minimum of Met-enkephalin in the ECEPP $/ 3$ force field as degenerated. However, the electrostatic energy for the ECEPP/3-minimum is raised substantially if ECEPP/2 charges are used for this structure. The total potential energy increases from $E_{t o t}=$ $-10.84 \mathrm{kcal} / \mathrm{mol}(\mathrm{ECEPP} / 3)$ to $E_{\text {tot }}=-9.46 \mathrm{kcal} / \mathrm{mol}(\mathrm{ECEPP} 2)$ while the energy of the other configuration changes only from $E_{t o t}=-10.81 \mathrm{kcal} / \mathrm{mol}(\mathrm{ECEPP} / 3)$ to $E_{t o t}=-10.72 \mathrm{kcal} / \mathrm{mol}(\mathrm{ECEPP} / 2)$, which is the global minimum of Met-enkephalin in the ECEPP/2 force field [25, 26]. Configurations of both group " 1 " and "2" were also found in our ECEPP/2 simulation.

We remark that our peptide seems to have a much richer spectrum of low energy local minimum configurations in the ECEPP/3 force field than in the case of ECEPP/2, but show in Fig. 3b only one more (the other contribute with less than $1 \%$ each) group. 
Conformers of this group (" $\mathrm{H}$ ") are characterized by a hydrogen bond between $\mathrm{C}=\mathrm{O}$ group of the $\operatorname{Tyr}(1)$ and the $\mathrm{N}-\mathrm{H}$ group of Met(5) residues. Together with the backbone dihedrals of Gly(2), Gly(3) and Phe(4) this hydrogen bonding resembles that of an $\alpha$ helix. Its lowest energy exponent has a potential energy of $-10.56 \mathrm{kcal} / \mathrm{mol}$ which is only $0.25 \mathrm{kcal} / \mathrm{mol}$ higher than the groundstate. No such conformation was found in the ECEPP $/ 2$ simulation. In the case of ECEPP $/ 2$, two more groups appeared with a frequency of more than $1 \%$ and are shown in Fig. 3a. Both are II' type $\beta$-turns. Group "a" is characterized by single hydrogen bond between Gly(2) and Met(5). The lowest energy conformation in this group has a potential energy of $-9.74 \mathrm{kcal} / \mathrm{mol}$ which make this group to the one with the second lowest potential energy, separated by $\approx 1 \mathrm{kcal} / \mathrm{mol}$ from the groundstate. Group "b" has its hydrogen bond between $\operatorname{Tyr}(1)$ and Phe(4), its lowest energy exponent has a potential energy of $-9.52 \mathrm{kcal} / \mathrm{mol}$ ). Both groups were also found in the ECEPP/3 simulation, but with frequencies less than $1 \%$ of the non-identical conformers.

For ECEPP/2 our local minimum conformations resemble those found in previous work [27] where in difference to the present work the peptide angles were released. Fixing these angles therefore seems to be a reasonable approximation. But while our classification of low energy local minima can also be obtained with other methods, the multicanonical approach allows in addition the estimation of the relative weight of these conformers as a function of temperature, i.e. entropic contributions can be considered. As an example we show in Fig. 5a and 5b the relative weight of the dominant groups of conformation for both ECEPP/2 and ECEPP/3. Note that around $300 K$ the percentage of configurations which are in none of our groups (and hence do not belong to the important valleys) decreases dramatically for both force fields. At high temperatures coil structures dominate which show large flexibility and and therefore a broad energy spectrum. On the other hand, at low temperatures ordered structures are expected which are each confined to one of the valleys in the energy landscape. In the case of the ECEPP/2 simulation the percentage of configurations of type ' 1 ' (the ECEPP/3 groundstate) never exceeded 4\%. Configurations of type '2' (the groundstate for ECEPP/2) are found with much higher probability, but at room temperature they contribute to only about $30 \%$ of the 
conformers which confirms older work [19]. For ECEPP/3 we find that the probability of configurations of type ' 2 ' is comparable to that of the ECEPP/2 simulation. At room temperature they contribute again to around $30 \%$ of the conformers. Conformations of type ' 1 ' appear with much higher probability than in the ECEPP/2 simulation, but that of type '2' are still dominating in the range of shown temperatures. At relevant temperatures the probability for finding the ECEPP/2 groundstate (type '2') is twice as high as that of finding the ECEPP/3 groundstate (type ' 1 '). Of course we expect that at $T=0 \mathrm{~K}$ we will find only the ECEPP/3 groundstate (type ' 1 '), however the energy difference is much too small (about $0.04 \mathrm{kcal} / \mathrm{mol}$ ) to favor them at finite temperatures against type '2' structures. This proofs that at least for small peptides entropic contributions cannot be neglected. Any pure optimization method (in the potential energy) may lead to configurations which are of only limited significance at relevant temperatures.

In the final plots we like to show how the variations in the force field affects two important thermodynamic quantities. Fig. 6 displays the average energy $\langle E\rangle$ as a function of temperature. Again, we observe only little differences between the ECEPP/2 and ECEPP/3 force fields for the physical relevant temperature range. Below $400 \mathrm{~K}$ these differences are within the errorbars, while for higher temperatures the ECEPP/3 energies are systematically lower by a small amount. Similar results were found for the specific heat $C(T)$ as function of temperature shown in Fig. 7. Here we define

$$
C(\hat{\beta})=\hat{\beta}^{2} \frac{<E^{2}>-<E>^{2}}{5} .
$$

Neither the position of its maximum nor its shape or heights vary much indicating that the transition between ordered and disordered states is of the same kind for both variants of the force field. Note that the position of the peak in specific heat corresponds to the increase of structures which belong to one of the main groups and the decrease of configurations which do not belong to any of the significant energy valleys.

\section{Conclusion}

We used the multicanonical approach to study variations of the energy landscape of Metenkephalin under a change from the ECEPP/2 force field to ECEPP/3. In both cases, the 
low-energy local minima were sampled and classified into a small number of groups which correspond to essential valleys of the energy landscape. We studied their distribution and relative weight at various temperatures. While the energy landscape differs in detail and specially the global minimum is not the same, our physical results are little affected by changes between the two force fields. Our analysis demonstrates that at least for small peptides it is not sufficient to search only for the global minimum in potential energy since this conformation may be of limited significance at room temperature. Our work corroborates the well known fact that small flexible molecules exist at room temperature in an ensemble of low energy conformations (see for instance Ref. [1] and work cited therein) whose relative weight has to be determined. Finally, our results show that numerical simulations of peptides are stable under small changes in the utilized force fields. Especially thermodynamic quantities are little changed. This is an important observation since all force fields rely on experimentally determined parameters and are therefore known only within certain errorbars.

\section{Acknowledgements:}

This work was supported, in part, by the Schweizerische Nationalfonds (Grant 20-40'838.94) and by the U.S. Department of Energy (contract DE-FC05-85ER250000). The simulations were performed on the cluster of RISC workstations at SCRI, The Florida State Univer-

sity, Tallahassee, USA, and the Institute for Biochemistry at the Humboldt University, Berlin, Germany.

\section{References}

[1] M. Vasquez, G. Nemethy and H. Scheraga, Chem. Rev. 1994, 94, 2183.

[2] S.J. Weiner, P.A. Kollman, D.T. Nguyen and D.A. Case, J. Comp. Chem. 1986, 7, 230. 
[3] B.R. Brooks, R.E. Bruccoleri, B.D. Olafson, D.J. States, S. Swaminathan and M. Karplus, J. Comp. Chem. 1983, 4, 187.

[4] F. A. Momany, R. F. McGuire, A. W. Burgess, and H. A. Scheraga, J. Phys. Chem. 1975, 79, 2361.

[5] G. Némethy, M. S. Pottle, and H. A. Scheraga, J. Phys. Chem. 1983, 87, 1883.

[6] M. J. Sippl, G. Némethy, and H. A. Scheraga, J. Phys. Chem. 1984, 88, 6231.

[7] G. Némethy, K. D. Gibson, K. A. Palmer, C. N. Yoon, G. Paterlini, A. Zagari, S. Rumsey, and H. A. Scheraga, J. Phys. Chem. 1992, 96, 6472.

[8] N. Metropolis, A. W. Rosenbluth, M. N. Rosenbluth, A. H. Teller, and E. Teller. J. Chem. Phys. 1953, 21, 1087.

[9] For a recent review see: S. Forrest, Science 1993, 261, 872.

[10] S. Kirkpatrick, C.D. Gelatt, Jr. and M.P. Vecchi, Science 1983, 220, 671.

[11] Z. Li and H.A. Scheraga, Proc. Nat. Acad. Sci. U.S.A. 1984, 84, 6611.

[12] U.H.E. Hansmann and Y. Okamoto, Numerical Comparison of Three Recently Proposed Algorithms in the Protein Folding Problem, ETH preprint TR-96-25, to appear in J. Comp. Chem.

[13] B. A. Berg and T. Neuhaus, Phys. Lett. 1991, B267, 249; B. A. Berg and T. Neuhaus, Phys. Rev. Lett. 1992, 86, 9.

[14] A. M. Ferrenberg and R. H. Swensen, Phys. Rev. Lett. 1988, 61, 2635; Erratum 1989, 63, 1658, and references given therein.

[15] B. Berg, U. Hansmann and T. Neuhaus, Phys. Rev. 1993, B47, 497; B. Berg, U. Hansmann and T. Neuhaus, Z. Phys. 1993, B90, 229.

[16] B. Berg and T. Celik, Phys. Rev. Lett., 1992, 69, 2292.

[17] B. Berg, T. Celik and U. Hansmann, Europhysics Letters, 1993, 22, 63; B. Berg, T. Celik and U. Hansmann, Physical Review 1994, B50, 16444. 
[18] T. Celik, U.H.E. Hansmann and M. Katoot, J. Stat. Phys., 1993, 73, 775.

[19] U. H. E. Hansmann and Y. Okamoto, J. Comp. Chem. 1993, 14, 1333.

[20] U.H.E. Hansmann, Y. Okamoto and F. Eisenmenger, Chem. Phys. Lett. 1996, 259, 321.

[21] M.H. Hao and H.A. Scheraga, J. Phys. Chem. 1994, 98, 4940.

[22] Y. Okamoto and U.H.E. Hansmann, J. Phys. Chem. 1995, 99, 11276.

[23] A. Kidera, Proc. Nat. Acad. Sci. U.S.A. 1995, 92, 9886.

[24] U. H. E. Hansmann and Y. Okamoto, Physica, 1994, A212, 415.

[25] Y. Okamoto, T. Kikuchi, and H. Kawai, Chem. Lett. 1992, 1275.

[26] H. Meirovitch, E. Meirovitch, A.G. Michel, and M. Vásquez, J. Phys. Chem. 1994, $98,6241$.

[27] B. von Freyberg and W. Braun, J. Comp. Chem. 1991, 12, 1065.

[28] The program SMC was written by F. Eisenmenger. 


\section{TABLE CAPTIONS:}

TAB. 1: Point Charges for Terminal Groups of Met-Enkephalin.

TAB. 2: Number of conformers and non-identical conformations collected after minimization for both choices of force fields.

TAB. 3: Dihedral angles of our estimates of the global minima for ECEPP/2 and ECEPP/3.

The peptide bond angles were fixed to $\omega=180^{\circ}$ in the simulations. 
Table 1:

\begin{tabular}{lrr} 
Atom & ECEPP $/ 2$ & ECEPP $/ 3$ \\
\hline \hline $\mathrm{N}$ & -0.356 & -0.332 \\
$\mathrm{H} 1, \mathrm{H} 2$ & 0.176 & 0.076 \\
\hline $\mathrm{C}$ & 0.450 & 0.517 \\
$\mathrm{O}$ & -0.384 & -0.351 \\
$\mathrm{O}$ & -0.380 & -0.334 \\
$\mathrm{H}$ & 0.204 & 0.235
\end{tabular}


Table 2:

\begin{tabular}{|l|l|l|}
\hline & ECEPP $/ 2$ & ECEPP/3 \\
\hline Minimized conformers $E<-6 \mathrm{kcal} / \mathrm{mol}$ & 62142 & 109863 \\
\hline $\begin{array}{l}\text { Non-identical minimized conformers } \\
\left(\geq 1 \text { dihedral differs by }>1^{\circ}\right)\end{array}$ & 1677 & 1574 \\
\hline
\end{tabular}


Table 3:

\begin{tabular}{|c|c|c|c|}
\hline & & ECEPP/2 & ECEPP/3 \\
\hline \multirow[t]{6}{*}{$\begin{array}{ll}1 & \text { Tyr }\end{array}$} & $\overline{\phi \phi}$ & -86.3 & $\overline{-162.7}$ \\
\hline & $\psi$ & 153.7 & -41.7 \\
\hline & $\omega$ & 180.0 & 180.0 \\
\hline & $\chi_{1}$ & -179.8 & -174.2 \\
\hline & $\chi_{2}$ & -111.4 & -85.2 \\
\hline & $\chi_{6}$ & 145.3 & 2.8 \\
\hline \multirow[t]{3}{*}{2 Gly } & $\phi$ & -161.5 & 65.8 \\
\hline & $\psi$ & 71.1 & -87.0 \\
\hline & $\omega$ & 180.0 & 180.0 \\
\hline \multirow[t]{3}{*}{3 Gly } & $\phi$ & 64.1 & -157.3 \\
\hline & $\psi$ & -93.5 & 34.9 \\
\hline & $\omega$ & 180.0 & 180.0 \\
\hline \multirow[t]{5}{*}{4 Phe } & $\phi$ & -81.7 & -158.8 \\
\hline & $\psi$ & -29.2 & 159.5 \\
\hline & $\omega$ & 180.0 & 180.0 \\
\hline & $\chi_{1}$ & 179.8 & 52.4 \\
\hline & $\chi_{2}$ & -100.0 & -96.0 \\
\hline \multirow[t]{7}{*}{5 Met } & $\phi$ & -80.7 & -82.4 \\
\hline & $\psi$ & 143.5 & 134.1 \\
\hline & $\omega$ & 180.0 & 180.0 \\
\hline & $\chi_{1}$ & -65.1 & -66.1 \\
\hline & $\chi_{2}$ & -179.2 & -179.6 \\
\hline & $\chi_{3}$ & -179.3 & -179.9 \\
\hline & $\chi_{4}$ & 60.1 & 60.1 \\
\hline$E_{t o t}(\mathrm{ECEEP} / 2)[\mathrm{kcal} / \mathrm{mol}]$ & & -10.72 & -9.46 \\
\hline$E_{t o t}(\mathrm{ECEPP} / 3)[\mathrm{kcal} / \mathrm{mol}]$ & & -10.81 & -10.85 \\
\hline
\end{tabular}




\section{FIGURE CAPTIONS:}

FIG. 1: Timeseries of potential energy, obtained from a multicanonical simulation of 200,000 MC sweeps, using a) the ECEPP/2 and b) the ECEPP/3 force field.

FIG. 2: Timeseries of clusters, obtained from a multicanonical simulation of 200,000 MC sweeps, using a) the ECEPP/2 and b) the ECEPP/3 force field.

FIG. 3: Most frequent low energy conformers for a) ECEPP/2 and b) ECEPP/3. Shown are also their Zimmermann codes, the frequency with which they appear in the multicanonical simulation and the potential energy of their lowest energy exponent $E_{\text {min }}$.

FIG. 4: Groundstate configurations of a) ECEPP/2 and b) ECEPP/3. The plots were drawn using RASMOL.

FIG. 5: Relative weight of the important groups of configurations in the case of a) ECEPP/2 and b) ECEPP $/ 3$. We have sum up all local minima which do not belong to one of the mayor groups in "Other". Likewise "No Minimum" contains all configurations which could not be minimized to a local minimum with potential energy below $-6 \mathrm{kcal} / \mathrm{mol}$ (see text).

FIG. 6: Average potential energy as a function of temperature for both choices of forcefields.

FIG. 7: Average of specific heat as a function of temperature for both choices of forcefields. 